\title{
An Ageing-aware Digital Synthesis Approach
}

\author{
Shengyu Duan, Basel Halak, Mark Zwolinski \\ School of Electronics and Computer Science, University of Southampton, UK \\ Email: $\{$ sd5g13, bh9, mz $\} @$ ecs.soton.ac.uk
}

\begin{abstract}
Due to the shrinkage of CMOS technology, wearout mechanisms such as Bias Temperature Instability (BTI) have raised growing concerns for circuit reliability. BTI can cause a threshold voltage shift in CMOS devices and consequently increase circuit delay. This paper presents an ageing-aware gatelevel optimization approach that can be used in a modern synthesis process. It aims to optimize a circuit to give improved lifetime reliability under given area and timing constraints. A new sensitivity metric is proposed as a function of area increase, delay reduction, degradation reduction and design constraints. This sensitivity metric can be adjusted to select the most favourable gates in terms of circuit timing, lifetime or both. By iteratively up-sizing the gates with the highest sensitivity, our proposed optimization flow can meet any realizable area and timing constraints, to give up to $3.3 \times$ lifetime improvement.
\end{abstract}

\section{INTRODUCTION}

As CMOS technology rapidly downscales, time-dependent transistor degradation has posed a great challenge to circuit reliability [1]. One of the most common wearout mechanisms, Bias Temperature Instability (BTI), manifests itself as an increase in transistor threshold voltage $\left(V_{t h}\right)$. For combinational circuits, BTI-induced $V_{t h}$ shift $\left(\Delta V_{t h}\right)$ results in path delay increase, eventually causing timing violations [2].

A practical approach to improve circuit lifetime at the design stage is to leave additional timing margins [3], which would increase the overheads of area and power. Thus, the circuit is over-designed. Alternatively, other work has proposed slowing down the actual degradation speed. A methodology to manipulate BTI stress by applying pin reordering and logic restructuring is presented in [4]. In [5], an iterative procedure is demonstrated to select and resize selected gates by considering BTI. Although these techniques can improve the lifetime reliability, the costs, in terms of other circuit characteristics like delay and area, are not really controllable. Thus an unacceptable overhead might be induced, especially for a design with significant constraints. None of these works has compared the performance penalties of their approaches with that caused by a conventional over-design technique.

In this work, we present a gate-level optimization approach to mitigate BTI degradation for given constraints. Specifically, we propose a new sensitivity metric that can be adjusted according to the design specifications, to pick the most favourable gate-level transformation in terms of timing, lifetime or both. In addition, an optimization flow employing the proposed sensitivity metric and gate up-sizing technique is demonstrated to mitigate BTI and satisfy specific timing and area constraints. We show that our strategy can be used as an alternative to over-design by having less area cost.
This paper is organized as follows. Section II describes the theory of BTI and degradation evaluation. Section III presents the proposed sensitivity metric and optimization algorithm. In Section IV, we show the results of our approach on some circuits. Finally, the paper is concluded in Section V.

\section{BTI EFFECT AND DELAY DEGRADATION}

\section{A. BTI Modelling}

According to the Reaction-Diffusion (R-D) theory, BTI can be physically described as the consequence of charge generation on the transistor oxide interface [6]. The charges are produced while the transistor is stressed (i.e. turned on), and will be partially neutralized in the OFF state. Thus these charges accumulate over time. The ratio of stressed time to the total is known as the stress duty cycle, which also indicates the signal probability $(S P)$ of a logic one/zero at a NMOS/PMOS transistor in a digital circuit.

A simplified BTI model is presented in [2] to quantify the delay shift $\left(\Delta D_{b t i}\right)$ for a given set of stress duty cycles and operational times as follows:

$$
\Delta D_{b t i}=K \cdot D_{0} \cdot \alpha^{n} \cdot t^{n}
$$

where $K$ is a constant parameter dependent on the technology node, temperature and supply voltage; $\alpha$ is the stress duty cycle; $t$ represents the operational time; $n$ is the time exponential constant equal to 0.16 [2]; and $D_{0}$ is the intrinsic delay.

Note that the proposed techniques in the remainder of this work do not necessarily rely on above model. Models proposed in other works like [7], are also compatible, since the trends in degradation will still hold.

\section{B. BTI Evaluation on Path Delay Degradation}

According to Equation 1, BTI is workload dependent (i.e. SP). Therefore, in order to evaluate path degradation due to BTI, one needs to capture the correct workload characteristics. Unfortunately, most previous work assumes that, for simplicity, the $S P$ at each primary input is $50 \%$ [3], [4]. This assumption fails to take the unpredictability of data inputs into consideration, for instance in a general purpose processor. The results may therefore not be accurate.

For a modern digital circuit with a large number of primary inputs, it is impractical to apply all the combinations of input $S P$ s, as the number is vast. Nevertheless, Bian et al, [8], applied a finite number of $S P \mathrm{~s}$ to all primary inputs, and showed that the overall path delay degradation has a Gaussianlike distribution. The Central Limit Theorem under weak dependence [9], suggests that the sum of an infinite number 
of weakly-dependent variables tends to have a Gaussian distribution. This condition is generally true for a combinational path: the total delay degradation is the sum of $\Delta D_{b t i}$ for all transistors constructing the path, and the dependence of $\Delta D_{b t i}$ on two transistors far enough apart from one another is negligible. In fact, path degradations can only approximate a Gaussian distribution, since a real path consists of finite transistors. But path degradations for all combinations of $S P$ should be generally convergent in a limited interval.

Motivated by this assumption, a dataset consisting of 2,500 combinations of $S P$ to all primary inputs was used to evaluate the path degradations. The size of the dataset, 2,500, was found in [8] to result in as consistent a distribution as those with more samples. We denote the mean of the distribution as the nominal path degradation $\left(\Delta D^{\text {nominal }}\right)$. Figure 1 shows $\Delta D^{\text {nominal }}$ due to Negative BTI (NBTI) for the critical path of an int to float converter from the EPFL combinational benchmark suite, [10]. The overall shape approximates a Gaussian distribution, if we consider both NBTI and Positive BTI (PBTI). The circuit is constructed from standard cells of a 65-nm technology node.

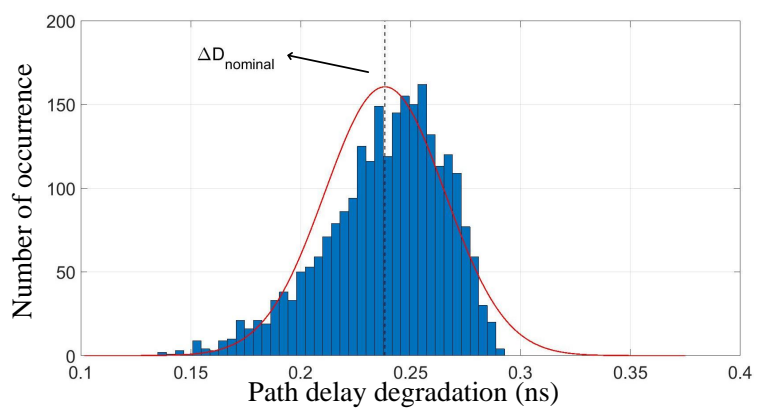

Fig. 1. Nominal case critical path degradation in int2float converter

\section{Proposed BTI-AWARE GATE-LEVEl Optimization}

\section{A. Problem Formulation}

Gate-level optimization in a modern synthesis process is performed as the final optimization phase following logic-level optimization and technology mapping. It attempts to meet the exact timing/area constraint by selecting the optimal cells from the library [11]. Nevertheless, current optimization techniques in commercial synthesis tools are blind to ageing effects. Thus, one can only over-design the circuit by putting in pessimistic timing margins to compensate for the impact of ageing.

The technique proposed in this work applies one of the most commonly used gate-level optimization transformations - gate up-sizing, but other techniques like load isolation are also applicable. The objective is not only to meet the design demand for timing and area, but also to minimize the maximal $\Delta D^{\text {nominal }}$ in the entire circuit. Thus we can formulate the problem for a circuit with $N$ paths as follows:

$$
\begin{array}{ll}
\text { Minimize } & \Delta D_{\max }=\max _{1 \leq i \leq N}\left(\Delta D_{i}^{\text {nominal }}\right) \\
\text { Subject to } & D_{\max }=\max _{1 \leq i \leq N}\left(D_{i}\right) \leq D_{\text {cons }} \\
& A \leq A_{\text {cons }}
\end{array}
$$

where $D_{i}$ and $D_{i}^{\text {nominal }}$ denote the intrinsic delay and delay degradation of path $i ; D_{\max }$ and $A$ indicate the maximal delay and total area of current design; $D_{\text {cons }}$ and $A_{\text {cons }}$ represent timing and area constraints respectively.

\section{B. A Constraint-related Sensitivity}

Delay-area sensitivity $\left(S_{D}\right)$, represented by the fraction of delay reduction over area increase, is used as a standard metric for gate sizing in commercial synthesis tools [12]. The transformation with the biggest delay-area sensitivity is performed until the timing constraints are met. The optimization aims to add as small as possible area to satisfy the timing specifications. While this technique does not account for the impact of BTI, degradation-area sensitivity $\left(S_{\Delta D}\right)$ is proposed to identify the transformation that can reduce the greatest degradation per unit area increase [5]. Since the degradation is proportional to the intrinsic delay according to Equation 1, upsizing the gate with the highest $S_{\Delta D}$ would also be favourable for circuit delay reduction. Thus the timing constraint would be met eventually, with the cost of more area.

Obviously, the circuit area is not really controllable by using either of the above sensitivities. We therefore propose a new sensitivity metric combining both to realize any customized area/timing constraints, as given by:

$$
\begin{aligned}
& S_{\text {prop }}=C_{\text {cons }} \cdot w \cdot S_{\Delta D}+S_{D} \\
& \text { where } \\
& C_{\text {cons }}=\max \left(0, \frac{\frac{A_{\text {cons }}}{A}-1}{1-\frac{D_{\text {cons }}}{D_{\text {max }}}}\right)
\end{aligned}
$$

and $C_{\text {cons }}$ is a constraint-related coefficient. $w$ is a weight, indicating the influence of $S_{\Delta D}$ on the proposed sensitivity, $S_{\text {prop. }}$. The value of $w$ is adjustable to satisfy any given constraints, as described in next subsection.

For better understanding of Equation 3, we analyse the characteristics of $S_{\text {prop }}$ under three scenarios:

Scenario 1: Consider a circuit with area $A$ very close to or bigger than $A_{\text {cons }}$, while the delay $D_{\max }$ is much greater than $D_{\text {cons }}$. In such a case, $S_{\text {prop }}$ is dependent mainly on $S_{D}$, since $C_{\text {cons }}$ tends to be zero. This situation can be interpreted as follows: if little area is left to accomplish a relatively big delay decrease, the optimization should be dedicated to reducing the delay and inducing as small an area increase as possible;

Scenario 2: Consider another extreme case, where $D_{\max }$ nearly reaches $D_{\text {cons }}$ but the difference between $A$ and $A_{\text {cons }}$ is big. Then $C_{\text {cons }}$ tends to infinity, and $S_{\text {prop }}$ is thereby determined by $S_{\Delta D}$. This case indicates the remaining area should be used to mitigate the degradation, if it is more than sufficient to meet the timing constraint;

Scenario 3: If neither of the above holds, $C_{\text {cons }}$ is a finite number. Thus $S_{\text {prop }}$ is determined by both $S_{D}$ and $S_{\Delta D}$, representing both delay and degradation in the transformation.

In summary, we can rewrite Equation 3 as follows:

$$
S_{\text {prop }}= \begin{cases}S_{D}, & \frac{A_{\text {cons }}}{A}-1 \ll 1-\frac{D_{\text {cons }}}{D_{\max }} \\ C_{\text {cons }} \cdot w \cdot S_{\Delta D}, & \frac{A_{\text {cons }}}{A}-1 \gg 1-\frac{D_{\text {cons }}}{D_{\text {max }}} \\ C_{\text {cons }} \cdot w \cdot S_{\Delta D}+S_{D}, & \text { otherwise }\end{cases}
$$




\section{Optimization Algorithm for BTI Minimization}

Figure 2 shows the proposed optimization algorithm to minimize BTI degradation under given constraints. Firstly, the weight $w$ from Equation 4 is initialized to a finite number. The initial value of $w$ does not really matter, since it can be adjusted in the remaining steps. However, a greater $w$ indicates $S_{\Delta D}$ has a stronger influence on $S_{\text {prop }}$, and the gate with high $S_{\triangle D}$ is more likely to be up-sized. In such a case, the overall optimization would bring about less degradation but more area according to last subsection. Secondly, the critical path, where the path delay is $D_{\max }$, needs to be identified. In addition, because $S_{\text {prop }}$ can be determined by both $S_{D}$ and $S_{\Delta D}$, the path with a degradation equal to $\Delta D_{\max }$, or the ageing-critical path, is identified as well. Afterwards, $S_{\text {prop }}$ on all gates of the critical/ageing-critical path is computed, and the gate with the highest $S_{\text {prop }}$ is sized up. The above steps are repeated until $D_{\text {cons }}$ is met. Finally, we check whether $A_{\text {cons }}$ is satisfied. A pre-defined constant $\epsilon$ is used to restrict the acceptable range of area: if the actual area does not lie in $\left[A_{\text {cons }}-\epsilon, A_{\text {cons }}\right]$, $w$ would increase or decrease depending on exact value of $A$, and the design is restored to the initial state, where any gatelevel transformations have not yet been applied. A maximum number of iterations can be set to give reasonable runtime.

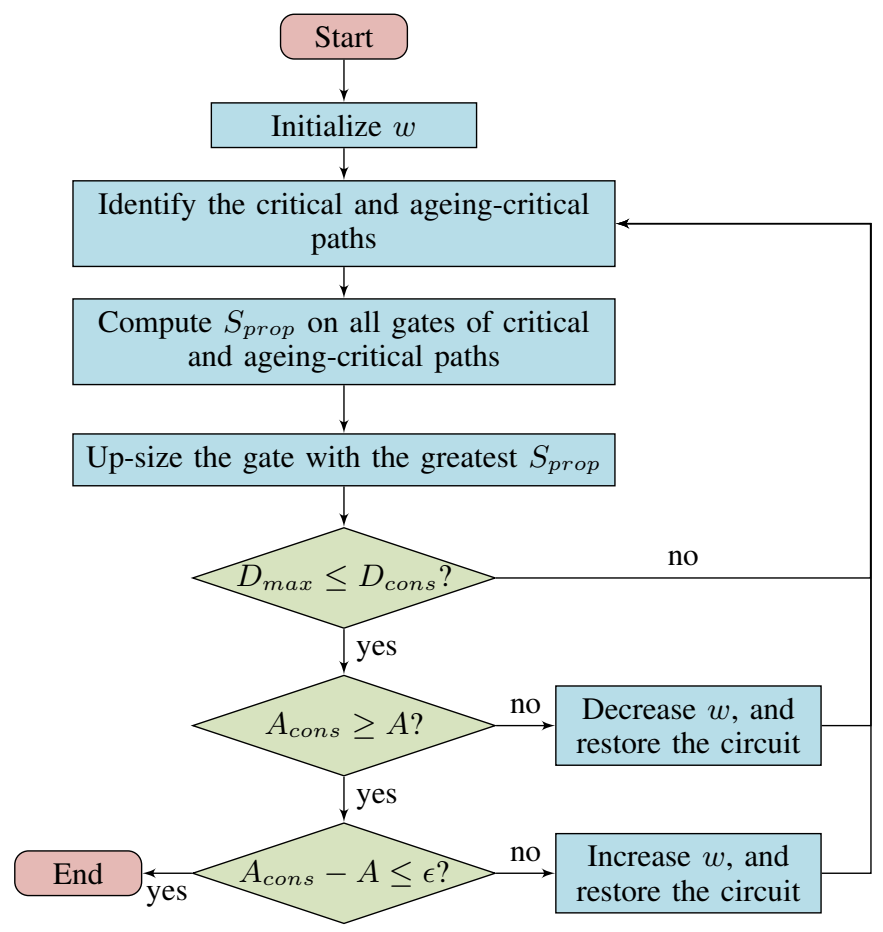

Fig. 2. The optimization flow to minimize BTI effect under given constraints

\section{EXPERIMENTAL RESULTS}

The proposed approach has been validated by applying it to several circuits from the EPFL combinational benchmark suite [10] for the case of NBTI only. For each circuit, we used Synopsys Design Compiler for logic-level optimization and our approach for gate-level optimization to optimize away the worst-case negative slack of 150ps. The area constraints were given by the equivalent gate count, with respect to the area of the smallest NAND cell. Referring to the three scenarios of Section III-B, we set three area constraints as follows:

1) $A_{\text {cons }}=0$, indicating using the least possible area as in Scenario 1 . This constraint is set as a standard to be compared with the two cases below, since $S_{D}$ is applied as a general metric in current synthesis tools. We denote the final circuit area in this case as $A_{\text {min }}$;

2) $A_{\text {cons }}=\infty$, meaning there is no upper limit for the area as in Scenario 2. The final area is denoted as $A_{\max }$;

3) $A_{\text {cons }}=A_{\text {cus }}$, where $A_{\text {cus }}$ represents the customized maximal allowed area. By definition, our approach can find the circuit with the optimal lifetime for any $A_{\text {cus }}$ within the range of $A_{\min }$ and $A_{\max }$. Here we show the case as $A_{\text {cus }}$ equalling to the average of $A_{\min }$ and $A_{\max }$, Scenario 3 .

Figure 3 shows the NBTI lifetimes of the int to float converter under the three area constraints after removing all negative slacks. As can be seen, the lifetime is extended as more area is used. Compared with the case of minimal area, the lifetime increases $184.48 \%$ and $252.95 \%$ for $A_{\text {cons }}$ are equal to $A_{\text {cus }}$ and $\infty$ respectively. This result indicates that the lifetime can be significantly extended by a relatively small amount of degradation reduction, because of the exponential behaviour of BTI degradation with respect to the operational time, as in Equation 1.

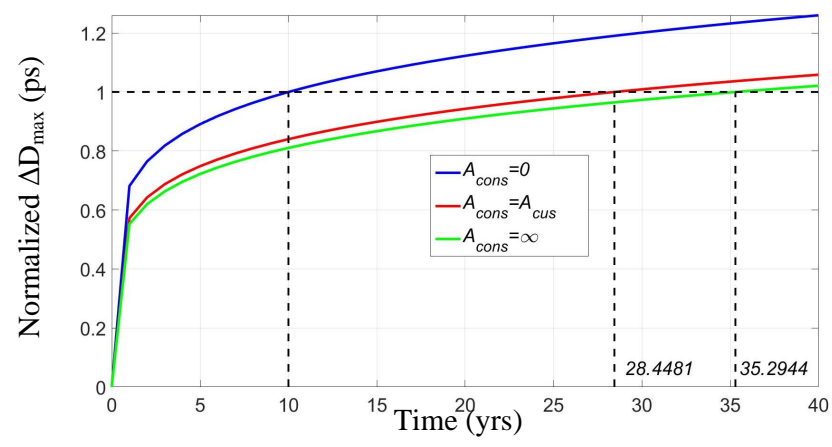

Fig. 3. Degradation changes and lifetime increases of int2float converter

Table I presents the optimization results for the experimental circuits. As can be seen, the minimal slacks of all circuits are positive, revealing that the timing constraints are satisfied by our proposed approach. In addition, the NBTI lifetime is optimized within the given amount of area overhead. For the case of $A_{\text {cons }}=0$, where the allowed area increase is too small to remove all negative slacks, the proposed approach can find the smallest design that satisfies the timing constraint. Comparing to the case of minimal area, the lifetime is improved by up to $337.48 \%$.

We compare the optimized circuits by our approach with the ones that are over-designed for the case of $A_{\text {cons }}=\infty$, in Table II. Equation 1 is used to compute the post-ageing delay. Assume both techniques can assure the circuits working 
TABLE I

CIRCUIT PERFORMANCES BY THE PROPOSED ALGORITHM (WORST SLACKS FOR ALL ORIGINAL CIRCUITS ARE -150PS)

\begin{tabular}{|c|c|c|c|c|c|c|c|c|c|c|c|}
\hline \multirow[t]{2}{*}{ Circuit } & \multicolumn{2}{|c|}{$A_{\text {cons }}=0$} & \multicolumn{4}{|c|}{$A_{\text {cons }}=\infty$} & \multicolumn{5}{|c|}{$\begin{aligned} A_{\text {cons }} & =A_{\text {cus }} \\
& =\left(A_{\min }+A_{\max }\right) / 2\end{aligned}$} \\
\hline & $\begin{array}{l}\text { Area } \\
\left(A_{\min }\right)\end{array}$ & $\begin{array}{l}\text { Min } \\
\text { slack } \\
(\mathrm{ps})\end{array}$ & $\begin{array}{l}\text { Area } \\
\left(A_{\max }\right)\end{array}$ & $\begin{array}{l}\text { Min } \\
\text { slack } \\
\text { (ps) }\end{array}$ & $\begin{array}{l}\text { Degradation } \\
\text { reduction } \\
(\%)\end{array}$ & $\begin{array}{l}\text { Lifetime } \\
\text { increase } \\
(\%)\end{array}$ & $A_{\text {cons }}$ & Area & $\begin{array}{l}\text { Min } \\
\text { slack } \\
\text { (ps) }\end{array}$ & $\begin{array}{l}\text { Degradation } \\
\text { reduction } \\
(\%)\end{array}$ & $\begin{array}{l}\text { Lifetime } \\
\text { increase } \\
(\%)\end{array}$ \\
\hline $\begin{array}{l}\text { Int to float } \\
\text { converter }\end{array}$ & 238.5 & 3.27 & 246.5 & 7.99 & 18.96 & 252.95 & 242.5 & 242.5 & 2.95 & 15.99 & 184.48 \\
\hline $\begin{array}{l}\text { Alu control } \\
\text { unit }\end{array}$ & 141.5 & 0.33 & 174.25 & 9.84 & 21.8 & 337.48 & 157.88 & 153 & 6.6 & 10.93 & 100.25 \\
\hline $\begin{array}{l}\text { Lookahead } \\
\text { XY router }\end{array}$ & 174 & 1.05 & 217.75 & 0.41 & 12.93 & 107.48 & 195.38 & 190.75 & 0.37 & 7.01 & 54.74 \\
\hline Coding-cavlc & 694.5 & 11.34 & 705.5 & 29.28 & 9.14 & 77.75 & 699.5 & 698.25 & 11.64 & 2.37 & 15.56 \\
\hline
\end{tabular}

properly in 10 years lifetime. $20 \%$ guardbands in terms of timing is required by conventional over-designing for a 65 $\mathrm{nm}$ technology from emperical study [3]. The guardbands are realized by gate-level optimization (i.e. incremental mapping) of Design Compiler. As can be noted, our approach can save $4.6 \%$ area on average to guarantee the same lifetime reliability. Thus, the proposed approach can work as an alternative to conventional over-design resulting in less area cost.

TABLE II

CIRCUIT PERFORMANCES OF OUR APPROACH AND OVER-DESIGNING

\begin{tabular}{|c|c|c|c|c|c|}
\hline \multirow{2}{*}{ Circuit } & \multicolumn{2}{|c|}{ Over-design } & \multicolumn{3}{|c|}{ Our approach } \\
\hline & $\begin{array}{c}10 \mathrm{yrs} \\
D_{\max } \\
(\mathrm{ps})\end{array}$ & Area & $\begin{array}{c}10 \mathrm{yrs} \\
D_{\max } \\
(\mathrm{ps})\end{array}$ & Area & $\begin{array}{l}\text { Area } \\
\text { saving } \\
(\%)\end{array}$ \\
\hline $\begin{array}{c}\text { Int to float } \\
\text { converter }\end{array}$ & 496.65 & 261.76 & 496.22 & 246.5 & 5.82 \\
\hline $\begin{array}{c}\text { Alu control } \\
\text { unit }\end{array}$ & 315.08 & 182.25 & 308.4 & 174.25 & 4.39 \\
\hline $\begin{array}{l}\text { Lookahead } \\
\text { XY router }\end{array}$ & 577.03 & 221 & 577.18 & 217.75 & 1.47 \\
\hline Coding-cavlc & 756.95 & 756.25 & 764.18 & 705.5 & 6.71 \\
\hline Average & & & & & 4.60 \\
\hline
\end{tabular}

\section{CONClusion}

In this paper, we present a novel gate-level optimization approach for digital synthesis considering ageing. Unlike stateof-the-art techniques, the objective of the proposed approach is not only to improve the lifetime reliability, but also to make sure the design is always within given specifications in terms of timing and area. A constraint-related sensitivity metric considering both delay and degradation reductions is applied. The sensitivity is adjustable to identify the most favourable transformation with regard to circuit timing, lifetime or both. Our optimization flow iteratively up-sizes the most favourable gate, which has the highest sensitivity according to the proposed metric. The results show for any realizable customized design specifications, our approach manages to find the design within the constraints, and with up to $337.48 \%$ lifetime improvement for the case of NBTI. By definition, this approach is also valid if considering both NBTI and PBTI. The cost of area of our approach is $4.6 \%$ less compared with a conventional over-design technique. Thus our approach is controllable and applicable to trade off between circuit delay, area and lifetime.

\section{ACKNOWLEDGEMENTS}

This work was supported by Cisco Research Center Grant No. 593688.

\section{REFERENCES}

[1] B. Kaczer, T. Grasser, J. Franco, M. T. Luque, P. Weckx, P. J. Roussel, and G. Groeseneken, "Assessing reliability of nano-scaled cmos technologies one defect at a time," in Emerging Electronics (ICEE), 2012 International Conference on. IEEE, 2012, pp. 1-2.

[2] K.-C. Wu and D. Marculescu, "Aging-aware timing analysis and optimization considering path sensitization," in Design, Automation \& Test in Europe Conference \& Exhibition (DATE), 2011. IEEE, 2011, pp. $1-6$.

[3] M. Ebrahimi, F. Oboril, S. Kiamehr, and M. B. Tahoori, "Aging-aware logic synthesis," in Proceedings of the International Conference on Computer-Aided Design. IEEE Press, 2013, pp. 61-68.

[4] K.-C. Wu and D. Marculescu, "Joint logic restructuring and pin reordering against nbti-induced performance degradation," in Proceedings of the Conference on Design, Automation and Test in Europe. European Design and Automation Association, 2009, pp. 75-80.

[5] A. Gomez and V. Champac, "A new sizing approach for lifetime improvement of nanoscale digital circuits due to bti aging," in Very Large Scale Integration (VLSI-SoC), 2015 IFIP/IEEE International Conference on. IEEE, 2015, pp. 297-302.

[6] A. Campos-Cruz, E. Tlelo-Cuautle, and G. Espinosa-Flores-Verdad, "Review: Advances in bti modeling for the design of reliable ics," in Electrical Engineering, Computing Science and Automatic Control (CCE), 2016 13th International Conference on. IEEE, 2016, pp. 1-4.

[7] E. Afacan, G. Dündar, A. E. Pusane, and F. Başkaya, "Semi-empirical aging model development via accelerated aging test," in Synthesis, Modeling, Analysis and Simulation Methods and Applications to Circuit Design (SMACD), 2016 13th International Conference on. IEEE, 2016, pp. $1-4$.

[8] S. Bian, M. Shintani, S. Morita, H. Awano, M. Hiromoto, and T. Sato, "Workload-aware worst path analysis of processor-scale nbti degradation," in Proceedings of the 26th edition on Great Lakes Symposium on VLSI. ACM, 2016, pp. 203-208.

[9] R. C. Bradley, "Central limit theorems under weak dependence," Journal of Multivariate Analysis, vol. 11, no. 1, pp. 1-16, 1981.

[10] L. Amarú, P.-E. Gaillardon, and G. De Micheli, "The epfl combinational benchmark suite," in Proceedings of the 24th International Workshop on Logic \& Synthesis (IWLS), no. EPFL-CONF-207551, 2015.

[11] Design compiler optimization reference manual, Synopsys Inc., 2010.

[12] D. G. Chinnery and K. Keutzer, "Linear programming for sizing, vth and vdd assignment," in Proceedings of the 2005 international symposium on Low power electronics and design. ACM, 2005, pp. 149-154. 